\title{
Influencia del medio de ensayo y dirección de aplicación de la carga en el comportamiento a corrosión bajo tensiones de la aleación de aluminio $7075^{(\cdot)}$
}

\author{
J.M. Badía*, J.M. Antoranz", P. Tarín*, A.G. Simón* y N.M. Piris* \\ Resumen El objetivo de este trabajo es comprobar la validez del empleo de agua de mar sintética \\ como método alternativo a la solución de $\mathrm{NaCl}$ utilizada en los ensayos de crecimiento de \\ grieta por corrosión bajo tensiones en aleaciones de aluminio, según la norma ASTM G- \\ 44. Para ello, se ha procedido al ensayo de muestras de aleación 7075 en diferentes estados \\ de tratamiento y con distintas orientaciones en ambos medios, comprobándose que pueden \\ considerarse equivalentes. Sólo las probetas ensayadas en la dirección del espesor mostraron \\ crecimiento de grieta durante los ensayos. La utilización del agua de mar representa una \\ gran ventaja práctica ya que altera mucho menos la superficie de las muestras y permite, por \\ tanto, una medida mucho más fiable de la grieta durante el ensayo.
}

Palabras clave Aleaciones de aluminio. Corrosión bajo tensiones. Ensayos. Crecimiento de grieta. Medio corrosivo.

\section{Influence of the corrosive medium and applied load direction on the stress corrosion cracking behaviour of 7075 aluminium alloy}

\begin{abstract}
The goal of this work is to prove the validity of using synthetic sea water as an alternative method instead of the $\mathrm{NaCl}$ solution used in stress corrosion crack growth tests in aluminium alloys, according to ASTM G 44. Several samples of 7075 alloy have been tested in different conditions and directions in both environments, which can be considered as equivalent. Only the samples tested in thickness direction have shown crack growth during the test. The use of sea water entails a great advantage in practice, by less superficial alteration, and lets therefore a much more reliable measure of the crack.
\end{abstract}

Keywords Aluminium alloys. Stress corrosion cracking testing. Crack growth. Corrosive media.

\section{INTRODUCCIÓN}

Las aleaciones de aluminio de alta resistencia utilizadas en la industria aeroespacial tienen tendencia a presentar, en determinadas condiciones, un proceso de corrosión bajo tensiones capaz de generar una grieta que, en el caso de no ser detectada a tiempo, podría desembocar, ocasionalmente, en un grave problema de seguridad de la aeronave ${ }^{[1]}$.

Por ello, antes de su empleo, las diferentes aleaciones y tratamientos a utilizar deben ser previamente investigados sobre su posibilidad de desarrollar este tipo de corrosión. Existen ensayos que permiten valorar de forma genérica su resistencia a la corrosión bajo tensiones, utilizando, por ejemplo, una probeta de tracción cargada a un cierto nivel de esfuerzos, en los que se determina el tiempo que tarda en romperse en un determinado medio corrosivo. Este tipo de ensayo valora de forma global el comportamiento a corrosión del material ensayado, incluyendo las etapas de nucleación de la grieta y su crecimiento, pero no permite determinar la velocidad a la que la grieta crece, y ese dato resulta importante en determinadas condiciones de diseño estructural.

Para determinar dicha velocidad de crecimiento existen otros ensayos diferentes en los que se crea, previamente, una grieta inicial, bien por

(•) Trabajo recibido el día 6 de julio de 2003 y aceptado en su forma final el día 3 de diciembre de 2004.

(*) Departamento de Materiales y Producción Aeroespacial. Escuela Técnica Superior de Ingenieros Aeronáuticos. Universidad Politécnica de Madrid. Ciudad Universitaria, 28040 Madrid. 
fatiga o por desgarro y, a continuación, se introducen en el medio agresivo para comprobar si dicha grieta crece y a qué velocidad lo hace. Para ello, la probeta más usualmente utilizada es del tipo DCB ${ }^{[2]}$.

En cuanto a los medios corrosivos utilizados, deben cumplir determinadas condiciones:

- Han de ser capaces de provocar la corrosión, en las aleaciones y estados susceptibles de presentarla, en un tiempo razonable de ensayo, muy inferior al que se precisaría para la aparición del problema en las condiciones reales de servicio.

- Deben ser capaces de discriminar con suficiente claridad entre las diferentes aleaciones, en función de su mayor o menor tendencia a la corrosión bajo tensiones.

- En la medida de lo posible, no deben facilitar el desarrollo de otros procesos simultáneos de corrosión que pudiesen interferir con la corrosión bajo tensiones.

La norma ASTM G-47 ${ }^{[3]}$, para determinar la susceptibilidad a corrosión bajo tensiones de aleaciones de aluminio de alta resistencia, indica que el medio de ensayo debe ser una solución al 3,5 \% de $\mathrm{NaCl}$ en agua destilada. En ella, debe hacerse una inmersión alternativa de las muestras a ensayar de acuerdo con lo recogido en la norma ASTM G-44 ${ }^{[4]}$, que especifica un ciclo de 10 min de inmersión de la probeta en la solución salina y 50 min de permanencia fuera de la misma para su secado.

Dicho medio produce durante el ensayo, según se recoge en la bibliografía ${ }^{[3-6]}$ (y ello se corresponde con la experiencia de este equipo investigador), una doble problemática que afecta seriamente al desarrollo del mismo y los resultados obtenidos:

- Se origina sobre la superficie de la muestra la deposición progresiva de una capa de productos de corrosión que dificultan o, incluso, impiden la correcta medición del tamaño de la grieta en los ensayos para determinar su velocidad de crecimiento, lo que obliga a retirarla antes de medir.

- Se desarrolla un proceso, más o menos acentuado, de ataque por picaduras, que puede interferir notablemente con el proceso de corrosión bajo tensiones, modificando el nivel local de las tensiones y colaborando en una más rápida fractura de la muestra en el caso de ciertos tipos de ensayos.
Por ello, a pesar de lo interesante que es ese medio para evaluar la resistencia a la corrosión bajo tensiones de estas aleaciones, se ha tratado de buscar alternativas que, de alguna manera, produjesen situaciones y respuestas del material similares, pero que no conllevasen dichos dos inconvenientes. Los nuevos medios de ensayo deberían ser contrastados con la clásica disolución de $\mathrm{NaCl}$, con vistas a comprobar que permiten diferenciar y clasificar de la misma manera a las diferentes aleaciones y tratamientos respecto de su susceptibilidad a la corrosión bajo tensiones. Desde este punto de vista, lo ideal sería utilizar un medio de ensayo que no altere superficialmente las probetas y que origine unos resultados lo más coincidentes posibles con los que se obtienen ensayando en las mismas condiciones con la disolución de $\mathrm{NaCl}$.

Dentro de esa línea, T.S. Humphries y E.E. Nelson $^{[5]}$ ya propusieron, en 1973, como posible alternativa, la utilización como medio corrosivo del agua de mar sintética, reproduciendo en laboratorio su composición de acuerdo con la norma ASTM D-114 ${ }^{[7]}$, excepto en elementos pesados. La elección de dicha disolución parecía razonable ya que, de hecho, representaba de alguna manera el ambiente real de servicio en que, en ciertos casos (aeropuertos junto al mar o portaaviones), se encuentran las aeronaves. Realizaron ensayos de corrosión bajo tensiones sobre una gran variedad de aleaciones y tratamientos, a partir de la norma ASTM G-44 ya citada, en la solución habitual de $3,5 \%$ de $\mathrm{NaCl}$ indicada por la norma y en agua de mar sintética. El ensayo elegido fue el de probetas de tracción cargadas a distintos niveles de tensión, determinándose el tiempo transcurrido hasta rotura.

Por otro lado, L. Schra y J. Faber ${ }^{[6]}$ tratan, igualmente, de estudiar de forma comparativa el comportamiento de ambos medios, en esta ocasión, sobre la velocidad de crecimiento de grieta en probetas $\mathrm{DCB}$ con ensayos de inmersión alternativa pero en condiciones muy diferentes a las especificadas por la norma ASTM G-44, que es la utilizada por mayoría de investigadores.

El objetivo del presente trabajo es, precisamente, tratar de confirmar la posibilidad de sustitución de la tradicional solución salina por la de agua de mar sintética para los ensayos de crecimiento de grieta sobre probetas $\mathrm{DCB}$, en las condiciones exigidas por la norma G-44, que es la más utilizada para determinar y comparar las velocidades de crecimiento de diferentes aleaciones y tratamientos. Para que dicha sustitución sea posible, las 
Influencia del medio de ensayo y dirección de aplicación de la carga en el comportamiento a corrosión bajo tensiones de la aleación de aluminio 7075 J.M. Badía, J.M. ANTORANZ, P. TARÍn, A.G. Simón y N.M. Piris

velocidades de crecimiento medidas, para todas las direcciones de aplicación de la carga, deben ser coincidentes en ambos medios de ensayo.

\section{PROCEDIMIENTO EXPERIMENTAL}

\subsection{Material utilizado}

El material de partida utilizado para la realización de estos ensayos ha sido una plancha de $25 \mathrm{~mm}$ de espesor de la aleación de aluminio 7075 en estado T651. Su composición química se recoge en la tabla I.

\subsection{Tratamientos térmicos realizados}

A distintos trozos de $160 \times 155 \mathrm{~mm}$ de la plancha se les aplicó un tratamiento de solución a $475^{\circ} \mathrm{C}$, con permanencia a esa temperatura durante $2,5 \mathrm{~h}$, en un horno de circulación forzada de aire y posterior enfriamiento en agua. A continuación, se introdujeron en una estufa, igualmente con circulación de aire, para recibir la correspondiente maduración artificial, con el propósito de conseguir los siguientes tratamientos finales:

- T6 : solución + maduración a $120^{\circ} \mathrm{C}$, durante $24 \mathrm{~h}$

- T76: solución + maduración de $4 \mathrm{~h}$ a $120^{\circ} \mathrm{C}+$ $17 \mathrm{~h}$ a $163^{\circ} \mathrm{C}$

- T73: solución + maduración de $7 \mathrm{~h}$ a $107^{\circ} \mathrm{C}+$ 26 h a $163^{\circ} \mathrm{C}$.

\subsection{Probetas utilizadas en los ensayos}

De los diferentes trozos de plancha, con los tratamientos T6, T76 y T73 realizados, así como de la plancha en estado original, T651, se procedió a la obtención y fabricación de probetas tipo $\mathrm{DCB}$, de $25 \times 25 \times 139 \mathrm{~mm}$ de dimensiones y con diferentes orientaciones respecto de la plancha (Fig. 1).

La mayoría de las probetas fueron extraídas de la posición Z-X, pero algunos ensayos en estado $\mathrm{T} 651$ se realizaron, igualmente, en las $\mathrm{X}-\mathrm{Y}$ e Y-X, siendo X la dirección de laminación de la plancha.

Tabla I. Composición de la aleación 7075 (\% en peso)

Table I. Composition of the 7075 alloy (weight \%)

\begin{tabular}{cccccccc}
\hline Al & Zn & Mg & Cu & Cr & Mn & Fe & Si \\
\hline Base & 5,62 & 2,32 & 1,47 & 0,2 & 0,08 & 0,25 & 0,15 \\
\hline
\end{tabular}

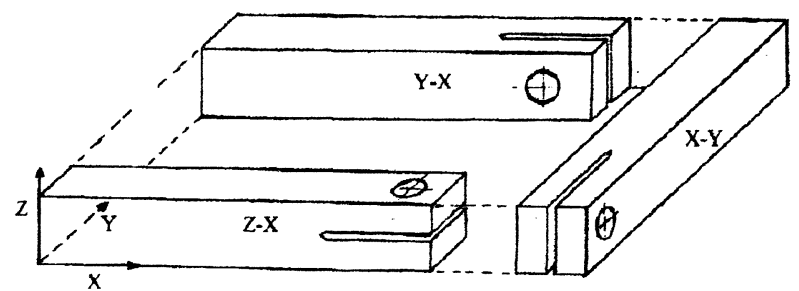

Figura 1. Orientación de las probetas en la plancha.

Figure 1. Direction of the specimens in the plate.

\subsection{Forma de realización de los ensayos}

La creación de la pregrieta inicial se realizó mediante desgarro, apretando uno contra otro los dos tornillos roscados en el extremo de la probeta en el que se mecanizó la entalla, hasta conseguir un tamaño de grieta tal que se distinguiese visualmente por los dos laterales de la muestra.

A continuación, las probetas fueron montadas en uná instalación que procedía a aplicar el ciclo de inmersión alternativa que prevé la norma G-44. Dicha instalación se encontraba en una habitación climatizada con $27 \pm 1{ }^{\circ} \mathrm{C}$ de temperatura y $45 \pm 2 \%$ de humedad relativa, de acuerdo, igualmente, con lo especificado en la citada norma.

Parte de las muestras fueron introducidas en una solución salina al 3,5\% de $\mathrm{NaCl}$ en agua destilada, según se indica en la norma G-44, mientras que otras lo eran en una disolución de agua de mar sintética, de acuerdo con lo indicado en ASTM D. 1141, sin metales pesados.

El tamaño de la grieta fue determinado midiendo la posición del frente de la misma mediante la técnica de ultrasonidos, utilizando un palpador de incidencia normal desde las dos caras de cada probeta. La duración total de los ensayos fue de $75 \mathrm{~d}$, realizándose medidas del tamaño de grieta en todas las muestras, cada semana.

\section{RESULTADOS}

\subsection{Aspecto superficial de las probetas}

A los pocos días de comenzar los ensayos de inmersión, empezó a detectarse un aspecto superficial muy diferente entre las probetas ensayadas en $\mathrm{NaCl}$ y en agua de mar. En las primeras, se iba formando una creciente capa de productos salinos, que impedía ver la grieta por el lateral de las probetas y obligaba a retirarla semanalmente en la zona en que se debía colocar el palpador para medir el tamaño de la grieta. Dicho ataque superficial se 
agravaba con el tiempo y daba lugar además al desarrollo de picaduras que entorpecían, aún más, la medición de la grieta.

Por el contrario, las probetas ensayadas en agua de mar no sufrieron ninguna deposición superficial significativa ni se desarrolló un apreciable ataque por picaduras, por lo que la grieta era siempre observable por los laterales y su medición mediante ultrasonidos no presentaba ninguna dificultad.

En la figura 2 puede apreciarse, claramente, la gran diferencia de aspecto superficial entre una probeta ensayada en solución de $\mathrm{NaCl}$ (recubierta de una capa de productos salinos) y otra sumergida en agua de mar (con su superficie prácticamente limpia).

\subsection{Crecimiento de grieta}

La tabla II recoge los crecimientos totales de grieta, medidos en las distintas probetas ensayadas se-
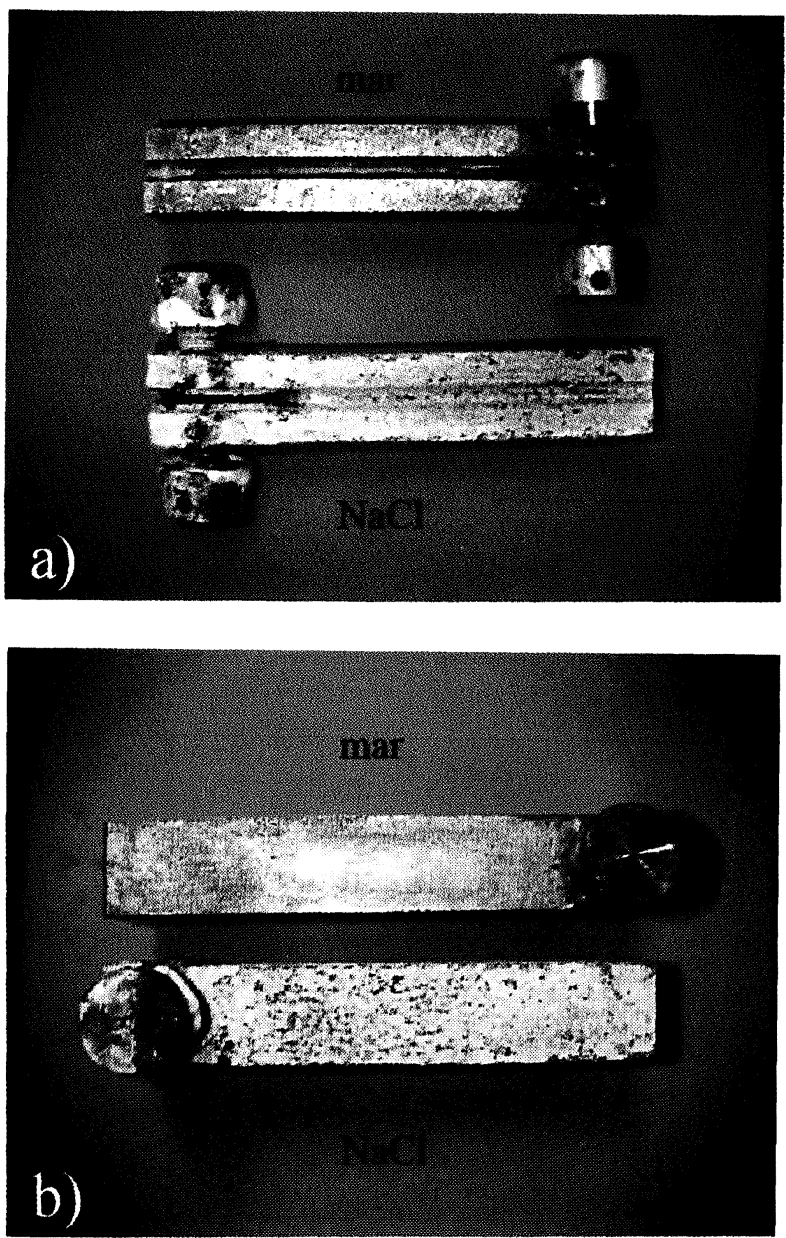

Figura 2. Aspecto superficial de las probetas $D C B$ en los medios usados: a) lateral, y b) superior.

Figure 2. Surface appearance of $D C B$ specimens in the tested environments: a) side view. b) upper view.
Tabla II. Crecimiento total de grieta en probetas DCB

Table II. Total crack growth on DCB specimens

\begin{tabular}{llll}
\hline Probeta & T. Térmico & Medio & $\Delta$ grieta $(\mathrm{mm})$ \\
\hline 1 & T651 & $\mathrm{NaCl}$ & 11,5 \\
2 & $\mathrm{~T} 651$ & $\mathrm{NaCl}$ & 12 \\
3 & $\mathrm{~T} 651$ & $\mathrm{mar}$ & 7,5 \\
4 & $\mathrm{~T} 651$ & $\mathrm{mar}$ & 10 \\
5 & $\mathrm{~T} 6$ & $\mathrm{NaCl}$ & 27 \\
6 & $\mathrm{~T} 6$ & $\mathrm{NaCl}$ & 50 \\
7 & $\mathrm{~T} 6$ & $\mathrm{NaCl}$ & 34 \\
8 & $\mathrm{~T} 6$ & $\mathrm{mar}$ & 64 \\
9 & $\mathrm{~T} 6$ & $\mathrm{mar}$ & 56,5 \\
10 & $\mathrm{~T} 76$ & $\mathrm{NaCl}$ & 0,5 \\
11 & T76 & $\mathrm{NaCl}$ & 5,5 \\
12 & T76 & $\mathrm{mar}$ & 1,5 \\
13 & $\mathrm{T76}$ & $\mathrm{mar}$ & 0,5 \\
14 & T73 & $\mathrm{NaCl}$ & 0 \\
15 & T73 & $\mathrm{mar}$ & 0 \\
\hline
\end{tabular}

gún la dirección del espesor (orientación Z-X), que es la más susceptible de presentar problemas de corrosión bajo tensiones ${ }^{[1]}$.

Se destaca, en ella, el muy diferente comportamiento apreciado en función del tratamiento térmico aplicado. El tratamiento T6, realizado en laboratorio, ha dado lugar a un gran crecimiento de la grieta, tanto en agua de mar como en $\mathrm{NaCl}$. El estado original T651 de la plancha, también se ha mostrado susceptible pero con menor agresividad. Por el contrario, los estados T7 han sufrido una pequeña o nula propagación de la grieta.

Si comparamos, para un mismo tratamiento, el comportamiento relativo de las probetas ensayadas en los dos medios utilizados, puede comprobarse que, para el estado T6, muy susceptible, el comportamiento de los dos medios ha sido similar, con crecimientos análogos en ambos. El bajo valor de propagación de las muestras 5 y 7 se explica porque, en ellas, la grieta se ha desviado mucho de la zona central de la probeta, lo que implica la existencia de un $\mathrm{K}_{\mathrm{I}}$ menor en el frente de grieta $\mathrm{y}$, por tanto, una propagación más lenta. Un efecto similar es referido, igualmente, en su trabajo por L. Schra ${ }^{[6]}$.

Para el estado original, T651, también con significativa susceptibilidad a la corrosión pero menor velocidad de crecimiento, los dos medios han producido crecimientos de grieta similares.

En el estado T76, el comportamiento en las dos soluciones vuelve a resultar similar, en principio, 
con la única anomalía de un crecimiento notablemente mayor en una de las muestras ensayadas en $\mathrm{NaCl}$.

Finalmente, ninguna de las probetas ensayadas, en estado T73, mostró señales de propagación de la grieta.

En cuanto a las probetas que se ensayaron en ambos medios, en estado T651, con orientaciones Y-X y X-Y, ninguna de ellas sufrió crecimiento de grieta durante los ensayos.

Por otro lado, para comparar el ritmo de crecimiento de la grieta en los dos medios corrosivos, se recoge (Fig. 3) el crecimiento de grieta en función del tiempo para las probetas 1 y 4, en estado T651, y las 6 y 8 , con tratamiento T6. Puede verse. en dicha figura que las curvas de crecimiento son bastante similares en los dos medios utilizados.

\section{DISCUSIÓN}

La observación del aspecto superficial de las probetas ensayadas en la solución de $\mathrm{NaCl}$ ha confirmado la experiencia previa existente, en el sentido de que se ha producido un progresivo deterioro superficial que dificulta, cada vez más, la medición de la grieta. Ello, coincide con lo comentado por L. Schra ${ }^{[6]}$ que indica, que si bien su programa de ensayos se organizó con una duración de 7 meses, las muestras ensayadas en $\mathrm{NaCl}$ debieron finalizar su ensayo tras dos meses, dada la imposibilidad de poder seguir midiendo la grieta con una lupa binocular por los laterales de las probetas, que era el método que utilizaba.

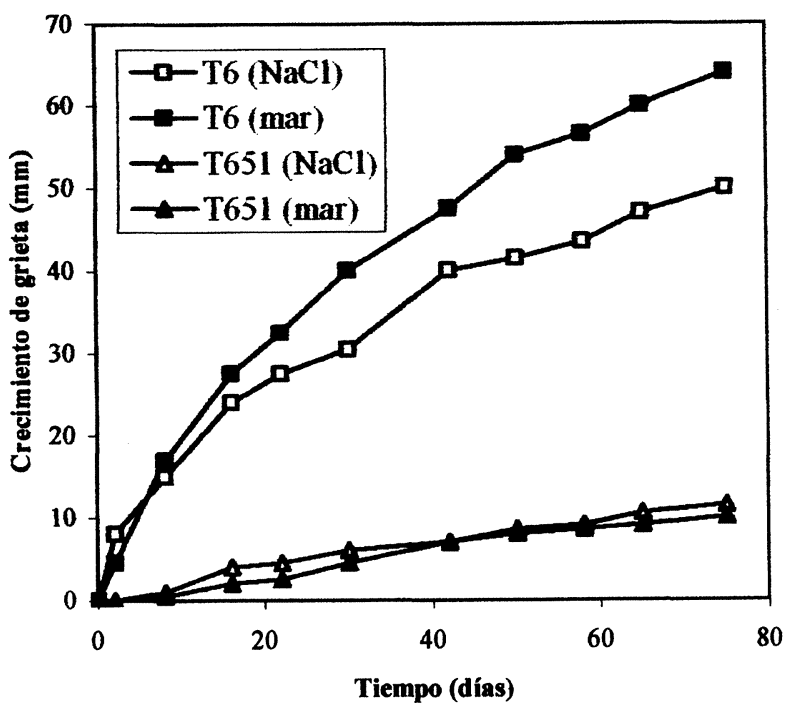

Figura 3. Crecimientos de grieta durante el ensayo.

Figure 3. Crack growths during the tests.
Por el contrario, en las probetas ensayadas en agua de mar no se produjo ningún deterioro significativo de su superficie, de manera que, incluso, tras los 2,5 meses de duración total de los ensayos, se podía posicionar fácilmente la grieta por ultrasonidos, sin necesidad de eliminar ninguna capa de productos de corrosión y localizar sin problemas la punta de grieta examinando los laterales de las probetas.

Las muestras en estado T651, ensayadas con las orientaciones X-Y e Y-X, en las que la tensión se aplicaba en las direcciones longitudinal y transversal-larga de la plancha, no han experimentado ningún crecimiento de la pregrieta inicial en ninguno de los medios. Esto, resulta coherente con la experiencia que se tiene sobre el comportamiento a corrosión bajo tensiones de productos laminados, en los que la resistencia a corrosión bajo tensiones es buena si la carga se aplica en esas direcciones, incluso para tratamientos térmicos que originan susceptibilidad al problema ${ }^{[1]}$.

Precísamente por ello, la mayoría de las probetas ensayadas lo han sido en dirección Z-X, con aplicación de la tensión en la dirección del espesor, en la que el comportamiento es mucho más desfavorable.

De los diferentes ensayos realizados en esta dirección, los correspondientes al estado T6 aplicado en nuestro laboratorio han sido los que han presentado un peor comportamiento a corrosión, con una velocidad de crecimiento de grieta muy notable. Las muestras ensayadas en estado original T651 han presentado, igualmente, una significativa susceptibilidad a corrosión, aunque el crecimiento de grieta ha sido más lento. Estos resultados coinciden con lo esperable ya que se considera que esta aleación, en estados de maduración T6, es bastante susceptible de presentar el problema bajo carga de tracción en la dirección del espesor ${ }^{[1]}$. La diferencia de comportamiento observada entre T6 y T651 puede estar relacionada con el hecho de que el T651 lleva, tras la etapa de solución, una pequeña deformación plástica por tracción, que relaja las tensiones internas.

Por otro lado, era de esperar, en principio, que los estados T7 proporcionasen una mayor resistencia a corrosión bajo tensiones, dado que, precisamente, esa es la razón fundamental de su empleo y que, entre ellos, el tratamiento T73, que conlleva una mayor sobremaduración, tuviese el mejor comportamiento. Estas expectativas se han cumplido en los ensayos realizados en ambos medios. El crecimiento de grieta detectado ha sido muy pequeño en estado T76 y nulo para las muestras en T73. 
En cuanto al comportamiento relativo de ambos medios de ensayo para los diferentes tratamientos, a la vista de los resultados obtenidos, parece deducirse que pueden considerarse, globalmente, como equivalentes, a la hora de valorar el comportamiento frente a la corrosión, sobre todo si el material presenta una alta susceptibilidad al problema.

Para situaciones de pequeña susceptibilidad, como la que se presenta en la aleación ensayada con el tratamiento T76, tal vez sería necesario realizar una mayor cantidad de ensayos para afirmar con rotundidad la equivalencia de ambos medios, dado que una de las muestras ensayadas en la solución de $\mathrm{NaCl}$ ha presentado un crecimiento significativamente mayor al del resto.

Estos resultados coinciden, en general, con los obtenidos por Schra y Faber ${ }^{[6]}$, utilizando probetas análogas pero condiciones de ensayo muy diferentes a las empleadas en este trabajo.

También coinciden con las conclusiones obtenidas por Humphries y Nelson ${ }^{[5]}$ que, incluso, afirman que el agua de mar es más interesante que la solución de $\mathrm{NaCl}$ para clasificar las aleaciones y sus tratamientos respecto de su resistencia a la corrosión bajo tensiones. Ahora bien, si se examinan con detenimiento los resultados que incluyen en su trabajo, realizado con ensayos muy diferentes a los aquí utilizados (por lo que los resultados no tienen por qué ser totalmente coincidentes), parece deducirse de ellos que, para aleaciones con pequeña susceptibilidad, el $\mathrm{NaCl}$ es más agresivo que el agua de mar.

\section{CONCLUSIONES}

- La solución de agua de mar sintética, utilizada como medio de ensayo para evaluar el creci- miento de grietas en probetas DCB mediante ensayos de inmersión alternativa, según la norma ASTM G-44, apenas altera la superficie de la probeta, lo que facilita enormemente la medición del tamaño de la grieta, frente al fuerte deterioro que se presenta en las muestras si se ensayan en una solución al 3,5\% de $\mathrm{NaCl}$.

- A la vista de los resultados obtenidos en probetas con distintos tratamientos (y en consecuencia con diferentes susceptibilidades a la corrosión bajo tensiones), parece que ambos medios producen efectos similares $\mathrm{y}$, por tanto, pueden considerarse equivalentes a la hora de evaluar el crecimiento de grieta con probetas DCB según la norma citada.

- En el estado T651, original de la plancha, únicamente se ha detectado crecimiento de grieta por corrosión en las probetas en las que la aplicación de las tensiones se hizo en la dirección del espesor, habiéndose comportado satisfactoriamente el material con cargas en las direcciones longitudinal y transversal-larga de la plancha.

\section{REFERENCIAS}

[1] ASM, Corrosion behavior, en Aluminum and Aluminum Alloys, ASM Specialty Handbook, 1993, Ohio, USA, 579. 622.

[2] A.J. SedRIKs, Stress Corrosion Cracking Test Methods, NACE, Houston, USA, 1990.

[3] ASTM, Norma G-47.

[4] ASTM, Norma G-44.

[5] T.S. Humphries y E.E. Nelson, NASA TM X-64733, 1973.

[6] L. Schra y J. FABER, NLR TR-81138-U, 1981.

[7] AsTM, Norma D-1141.

[8] L. Schra y R.J.W. WANHILl, Aluminium 3 (1983) 191-194. 